Apidologie, 1977, 8 (4), 357-361.

\title{
DIE KONZENTRATION UND ZUSAMMENSETZUNG VON NEKTAR IN BEZIEHUNG ZU BIENENBESUCHEN AN OBSTBÄUMEN
}

\author{
La concentration et la composition du nectar en fonction \\ des visites d'abeilles sur les arbres fruitiers
}

\section{J. MOMMERS,}

Proefbijenstand a Ambrosiushoeve v, Hilvarenbeek, Niederlande.

\author{
SUMMARY \\ THE CONCENTRATION AND COMPOSITION OF NECTAR IN RELATION \\ TO HONEYBEE VISITS TO FRUITTREES
}

Nectarsecretion and honeybee visits were compared to different apple and pear varieties. Nectar of pears has sometimes so little sugar, that the taste is not sweet for bees. Nectar of pear; contains only spur; of saccharose. Yellow Transparaat has more sugar in the nectar and is better visited than Boskoop. Statistically reliable a similar correlation could not be established between ths quantity of nectar and bee visits to Jonathan, Cox's Orange and Golden delicious. Concerning the percentage sugar in the nectar only to Golden delicious an equal correlation existed.

\section{ZUSAMMENFASSUNG}

Nektarabsonderung und Bienenbesuch bei verschiedenen Apfel-und Birnensorten wurden verglichen. Birnennektar hat manchmal so wenig Zucker, dass er für die Bienen nicht süss schmeckt. Birnennektar enthält nur Spuren Saccharose. Yellow Transparant hat mehr Zucker im Nektar und wird besser beflogen als Boskoop. Für die übrigen Apfelsorten konnte eine derartige Korrelation nicht statistisch zuverlässig festgestellt werden. Es gibt eine lineare Korrelation zwischen der Menge Nektar und dem Bienenbesuch bei Jonathan, Cox's Orange und Golden Delicious. Eine gleiche Korrelation hinsichtlich Prozentsatz Zucker im Nektar gab es nur bei Golden Delicious. 
Schon 1964 hielt ich beim 2. Internationalen Symposium über Bestäubung in London ein kurzes Referat — als vorläufige Mitteilung — über oben genanntes Thema.

Es war klar, dass, um sicherere Resultate zu bekommen, die Anzahl der Beobachtungen viel grösser sein musste. Die äusseren Umstände während der Obstblüte sind nämlich von Jahr zu Jahr und von Tag zu Tag sehr verschieden. Jedes Jahr gibt es nur wenige Tage, an denen Beobachtungen an blühenden Obstbäumen stattfinden können. Darum mussten diese viele Jahre fortgesetzt werden (bis 1971).

Die Beobachtungen betrafen : die Menge, die Konzentration und Zusammensetzung des Nektars und die Anzahl von Bienen, die die Blumen von verschiedenen Obstrassen besuchten.

Die Untersuchungen fanden statt an der Versuchsstation für Bienenzucht "Ambrosiushoeve ", wo im Obstgarten eine Anzahl gleich alter Obstbäume (Äpfel und Birnen) verschiedener Rassen stehen, alle auf die selbe Unterlage gepropft.

Zur Nektaruntersuchung wurden blühende Zweige eingehüllt und nach 24. Stunden wurde mit einer feinen Pipette der Nektar von 100 Blumen gesammelt. Da sich gezeigt hatte, dass die Nektarabsonderung oben und unten am Baum verschieden ist, wurden — soweit möglich — Zweige aus gleicher Höhe gewählt.

Jedesmal wurde von zwei Bäumen der selben Sorte der Nektar gesammelt. Der Zuckergehalt wurde refraktometrisch bestimmt und die im Nektar enthaltenen Zucker (Saccharose, Glukose und Fruktose) papierchromatografisch ermittelt.

Die blütenbesuchenden Bienen wurden gezählt in 5-Minuten- Perioden an möglichst genau 100 offenen Blumen (in den ersten 2 Jahren 100 Blütenbüscheln), während möglichst vieler Stunden pro Tag, abhängig vom Wetter. Auf diese Weise wurden viele Daten gesammelt und zwar so viele, dass das Ganze sehr unübersichtlich ist.

Das Ziel der Untersuchungen war, zu sehen, ob die Bienen Blumen mit mehr oder besserem Nektar bevorzugen. Wenn das der Fall ist, dann kann es nützlich sein, bei der Anlage von Obstanpflanzungen darauf zu achten. Die Kreuzbestäuber wären so zu wählen, dass der Nektar ungefähr gleichwertig ist.

Bevor ich aber die Resultate in diesem Sinne bespreche, möchte ich - weil ich von Nektaruntersuchung spreche - zuerst einige Bemerkungen über die Nektarabsonderung machen.

Im allgemeinen hat der Nektar bei Birnen einen geringeren Zuckergehalt als bei $\ddot{\text { Apfeln. }}$ 
Einige extreme Fälle : 1968 am 24. April, Conférence 918 mg Nektar - also ziemlich viel, mit 1,06\% Zucker; also pro 100 Blumen 9,70 mg Zucker. Noch grössere Mengen Nektar bei Conférence am 22. April 1968 : 1190 mg Nektar, aber 1,87\% Zucker. Am 25. April 1968 aber bei Beurré Hardy 800 mg Nektar, 13,47\% Zucker, also pro 100 Blumen 108,00 mg Zucker !

$1967 \mathrm{am}$ 24. April Triomphe de Vienne $7 \mathrm{mg}$ Nektar pro 100 Blumen, 50,10\% Zucker, pro 100 Blumen nur 3,50 mg Zucker. Obwohl viele Ausnahmen vorkommen, kann man doch wohl im allgemeinen sagen : Eine grosse Menge Nektar enthält verhältnismässig wenig Zucker und umgekehrt. Zuckerlösungen mit weniger als $3 \%$ Zucker werden von den Bienen nicht angenommen (v. Frisch).

Birnennektar enthält nur Spuren von Saccharose. Die Mengen von Glukose und Fruktose sind annähernd gleich. Vielleicht ist das auch eine der Ursachen, warum Bienen die Birnen im allgemeinen schwach befliegen.

Bei Äpfeln ist sowohl der Prozentsatz Zucker als die Zuckermenge pro 100 Blumen höher als bei Birnen. Es gibt hier aber auch sehr grosse Schwankungen. Die Menge Saccharose ist bei Äpfeln etwas mehr als Glukose und Fruktose zusammen. Die drei Zucker zusammen sind immer weniger als das Totalzuckergewicht.

Die Daten über Nektarabsonderung und Bienenbesuch sind statistisch bearbeitet worden von Herrn Ir. JANSEN vom Institut TNO voor Wiskunde, Informatieverwerking en Statistiek in Wageningen. Auf die Methodik werde ich hier nicht eingehen. Die Daten befinden sich bei J. J. Petringa, der sie gerne für Interessierte bereitstellt (gleiche Adresse wie Autor). Weitere Berechnungen wurden ausgeführt von Herrn Kerkviret. Die beobachteten Apfelsorten waren : Yellow Transparant (Klarapfel), Boskoop, Cox's Orange, Jonathan und Golden Delicious. Weil nicht alle Sorten zugleich blühen, wurden Paare mit derselben Blütezeit gebildet und zwar : Yellow Transparant-Boskoop, Cox's Orange-Jonathan, Cox's Orange-Golden Delicious und Jonathan-Golden Delicious. Bei diesen Paaren wurde berechnet, ob es statistisch gesicherte Unterschiede gibt, hinsichtlich mg Zucker in 100 Blumen und durchschnittlicher Zahl von Bienenbesuchen an 100 Blumen.

Schluss : Yellow Transparant wird besser beflogen als Boskoop und hat auch mehr Zucker im Nektar. Cox's Orange wird weniger von Bienen besucht als Jonathan. Zwischen der Nektarabsonderung bei beiden Sorten ist kein statistisch sicherer Unterschied.

Herr Kerkvliet bestimmte die durchschnittliche Menge Nektar in mg pro 100 Blumen und den durchschnittlichen Prozentsatz Zucker im Nektar und berechnete, ob es eine lineare Korrelation zwischen diesen und dem Bienenbesuch gibt (Tab. 1). 
Тав. 1. - Ergebnisse der statistischen Untersuchung.

TABL. 1. - Résultats des calculs statistiques.

\begin{tabular}{|c|c|c|c|c|c|}
\hline $\begin{array}{l}\text { ÄPFEL } \\
\text { POMMES }\end{array}$ & Boskoop & Jonathan & $\begin{array}{l}\text { Yellow } \\
\text { Transp. }\end{array}$ & Cox Oran. & G. Delicious \\
\hline $\begin{array}{l}\text { Mittelwert mg Nektar pro } 100 \\
\text { Blumen (a) . . . . . . . . . . } \\
\text { Quantité moyenne de nectar en mg } \\
\text { pour } 100 \text { fleurs (a) }\end{array}$ & $93,52 \mathrm{mg}$ & $114,85 \mathrm{mg}$ & $195,25 \mathrm{mg}$ & 174,53 & $172,43 \mathrm{mg}$ \\
\hline $\begin{array}{l}\text { Standardabweichung von a .. } \\
\text { Écart-standard de a }\end{array}$ & 101,42 & 69,81 & 91,18 & 104,40 & 189,68 \\
\hline $\begin{array}{l}\text { Mittelwert Bienenbesuch (b) } \\
\text { Valeur moyenne des visites d'a- } \\
\text { beilles (b) }\end{array}$ & 2,13 & 3,34 & 3,96 & 2,09 & 3,25 \\
\hline $\begin{array}{l}\text { Standardabweichung von } b \ldots \\
\text { Ecart-standard de b }\end{array}$ & 1,48 & 2,73 & 2,14 & 1,29 & 2,14 \\
\hline $\begin{array}{l}\text { Mittelwert } \% \text { Zucker im Nek- } \\
\text { tar (c) ... . . . . . . . . . . } \\
\text { Teneur moyenne en sucre du nectar } \\
\text { (c) }\end{array}$ & $45,27 \%$ & $47,37 \%$ & $32,94 \%$ & 36,44 & $4,4,09 \%$ \\
\hline $\begin{array}{l}\text { Standardabweichung von } c \ldots \\
\text { Écart-standard de } \mathrm{c}\end{array}$ & 17,73 & 16,65 & 15,40 & 18,54 & 17,36 \\
\hline $\begin{array}{l}\text { Korrelation von a und } b \ldots \ldots \\
\text { Corrélation entre a et b }\end{array}$ & 0,241 & $0,30^{2}$ & $0,14^{1}$ & 0,51 & $0,60^{3}$ \\
\hline $\begin{array}{l}\text { Korrelation von b und } c \ldots \\
\text { Corrélation entre b et } c\end{array}$ & $0,12^{1}$ & $0,10^{1}$ & 0,221 & $0,30^{1}$ & 0,50 \\
\hline $\begin{array}{l}\text { Anzahl Beobachtungen } \ldots . . \\
\text { Nombre d'observations }\end{array}$ & 25 & 32 & 19 & 23 & 23 \\
\hline $\begin{array}{l}\text { BIRNEN } \\
\text { POIRES }\end{array}$ & Beurré $\mathbf{H}$. & Confé & Tric & he d.V. & $\begin{array}{l}\text { Doyenné } \\
\text { du Comice }\end{array}$ \\
\hline $\begin{array}{l}\text { Mittelwert mg Nektar pro } 100 \\
\text { Blumen (a) . . . . . . . . . . } \\
\text { Quantité moyenne de nectar en mg } \\
\text { pour } 100 \text { fleurs (a) }\end{array}$ & $181,89 \mathrm{mg}$ & 236,4 & & $14 \mathrm{mg}$ & $313,96 \mathrm{mg}$ \\
\hline $\begin{array}{l}\text { Standardabweichung von a .. } \\
\text { Écart-standard de a }\end{array}$ & 254,46 & $\mathbf{3 4 8 , 5}$ & & & $\mathbf{3 7 1}, \mathbf{3 3}$ \\
\hline $\begin{array}{l}\text { Mittelwert Bienenbesuch (b) . } \\
\text { Valeur moyenne des visites d'abeil- } \\
\text { les (b) }\end{array}$ & 1,69 & 2,0 & & & 3,97 \\
\hline $\begin{array}{l}\text { Standardabweichung von } b \text {.. } \\
\text { Écart-standard de } b\end{array}$ & 1,24 & 1,5 & & & 1,24 \\
\hline $\begin{array}{l}\text { Korrelation von a und } b . \ldots \\
\text { Corrélation entre a et } b\end{array}$ & $0,15^{1}$ & 0,1 & & $10^{1}$ & $0,39^{1}$ \\
\hline $\begin{array}{l}\text { Anzahl Beobachtungen } \ldots \ldots \\
\text { Nombre d'observations }\end{array}$ & 16 & 19 & & & 14 \\
\hline
\end{tabular}

1. nicht signifikant.

2. signifikant mit Wahrseheinlichkeit von $95 \%$.

3. signifikant mit Wahrscheinlichkeit von $99,5 \%$.

4. signifikant mit Wahrscheinlichkeit von $99,0 \%$.

1. non significatif.

2. significatif à $95 \%$.

3. significatif à $99,5 \%$.

4. significatif à $99,0 \%$ 
Hinsichtlich der Menge Nektar war das der Fall bei Jonathan, Cox's Orange und Golden Delicious, hinsichtlich Prozentsatz Zucker nur bei Golden Delicious.

Bei Birnen wurde Beurré Hardy mit Conférence, Beurré Hardy mit Triomphe de Vienne, Doyenné du Comice mit Conférence und Doyenné du Comice mit Triomphe de Vienne verglichen.

Doyenné du Comice hat mehr Bienenbesuch als Conférence und Triomphe de Vienne, Triomphe de Vienne mehr als Beurré Hardy (weniger sicher). Zwischen den Paaren bestehen Unterschiede in der Nektar-Zuckermenge.

Bei Birnen konnte keine lineare Korrelation zwischen Nektarmenge und Bienenbesuch festgestellt werden. $\mathrm{Ob}$ es vielleicht eine andere Korrelation gibt, ist nicht untersucht worden.

Es sind also mit den bis jetzt gebrauchten statistischen Methoden keine allgemein gültigen Schlüsse zu ziehen.

Zum Schluss will ich aber noch eine Beobachtung, die nebenbei gemacht wurde, erwähnen : Wenn der Nesseltuchsack nach 24 Stunden beseitigt wurde, um den Nektar den Blumen zu entnehmen, konnte man das jedesmal nur schrittweise machen. Die Bienen flogen nämlich sofort massenhaft zu den nektarreichen Blumen. Sie sind offenbar imstande, eine bessere Trachtquelle sehr schnell zu finden und auszunützen.

Eingegangen im April 1977.

Rę̧u pour publication en avril 1977.

\section{RESUMÉ}

On a comparé la sécrétion nectarifère et les visites des abeilles sur différentes variétés de pommier et de poirier. Le nectar de poirier ne renferme que des traces de saccharose. La Yellow Transparant a un nectar plus sueré et est plus visitée que la Boskoop. On n'a pas pu établir de relation semblable, statistiquement significative, entre la quantité de nectar et les visites sur Jonathan, ' 'ox' orange et Golden delicious. Une telle relation concernant la teneur en sucre du nectar n'existe que pour la Golden delicious.

\section{LITERATUR}

JANsev A. A. M., 1974. - Nektaronderzoek; analyse van de gegevens. Institut TNO voor Wiskunde, Informatieverwerking en Statistiek, afd. Wegeningen. Rapport 10-7-1974.

Frisch K. von, 1927. - Versuche über den Geschmacksinn der Bienen. Die Naturwissenschaften, 15 (14).

Mommers J., 1966. - Die Nektarabsonderung bei verschiedenen Rassen der Obstbäume. Z. Bienenforsch., 8, 203-204.

Mommers J., 1966. - The concentration and composition of nectar in relation to honeybee visits to fruittrees. 2nd int. Symp. Pollin.tion. 1964. Bee World, 47 (1), suppl., 91-94. 